\title{
SIFT points for screening Based on random forest in INS/SAR system
}

\author{
Hao Dou ${ }^{1}$,ShaoJun $\mathrm{Li}^{2}$, Xiao Sun ${ }^{1}$,Tian Tian ${ }^{1}$, ShuiPing Zhang ${ }^{1}$, DeLie Ming ${ }^{{ }^{*}}$ \\ ${ }^{1}$ National Key Laboratory of Science and Technology on Multi-spectral Information Processing, \\ Huazhong University of Science and Technology, Wuhan 430074, China; \\ ${ }^{2}$ National Key Laboratory of Science and Technology on Aerospace Intelligence Control, Beijing Aerospace \\ Automatic Control Institute ,Beijing 100854 China;
}

Keywords: machine learning, matching point, random forest

\begin{abstract}
The paper adopts machine learning framework to analyze matching suitability of SIFT match points in INS/SAR integrated navigation system. The paper adopts machine learning framework to analyze matching suitability of SIFT match points in INS/SAR integrated navigation system. It aims to select the matching points for the feature matching steps in the SIFT algorithm. So as to improve the proportion of the correct matching points and then reduce the time-consuming of the RANSAC algorithm. This paper first extract the adaption features of the matching points. including the nearest distance, nearest and second nearest distance, the scale and so on, and models the constructing classifier using the random forest to complete the prediction of the unknown matching points.
\end{abstract}

\section{Introduction}

These years, SIFT points on screening study in INS/SAR integrated navigation system has been not very mature, in the original SIFT algorithm, the used method is just setting a simple artificial threshold to remove points, whose the nearest distance and the second nearest distance's ratio is relatively large. However, the matching point to filter is a multivariate effect problem, this method does not apply in the SAR images. In actual, Matching point's screening can be viewed as the machine learning's $0 / 1$ classification problem. Thus, this paper proposes adopting machine learning framework. At first, building matching point pairs adaptive feature, then, using training for random forest classifier, completing the matching points for filtering and prediction, after Screening, the the correct match ratio of the match points is improved, reducing the number of total matching points on matching so as to improve the performance.

\section{The adaptive feature extraction of Matching points}

Match points establish relationship between real-time image and benchmark image, this relationship can separate into two class. The first class mainly comes from SIFT key points, which includes scale, group number these information, as to the same location's Real-image and benchmark image, it is reasonable to require the information difference between them is relatively small, otherwise, it is believed that distortion of the area near the position is larger or unstable. The other class mainly measured by the descriptor of SIFT, which is similar to the related surface analysis of real-time image SIFT descriptor and benchmark image SIFT descriptor, including the matching points closest distance or the ratio between the closest distance and the second closest distance and so on. The experiment takes 15 airborne SAR and spaceborne SAR pairs respectively as scene matching Real-image and benchmark image, The resolution of which are both 10 meters. Count the relationship between adaptation of the matching point statistical characteristics and correlation matching accuracy, to illustrate the effectiveness of our adaptive characteristics.

\section{Screening matching points based on machine learning}

Screening matching points is a challenge problem, choosing good point pairs in advance is helpful for SAR scene matching. Using artificial rules to complete various screening is a more direct means, but it is too extensive. Screening SIFT matching points is a multivariate influence 
problem, so it needs a fine rule in the processing, however, its effect may be not good. The input of matching points screening is multidimensional eigenvectors, the output is marking the key point which is good or bad for matching using Boolean value or matching probability. This actually is a classification problem in pattern recognition.

We first select a number of points in the matching points data set as a training set, then, extracting corresponding matching feature and getting whether point is good for matching or not in the actual matching process and marking the point with land 0 , after that, training the model of training set to get the classifier. At last, we can use the classifier to predict whether the point is fit for matching or not, so we finish screening matching points using Supervised learning in machine learning.

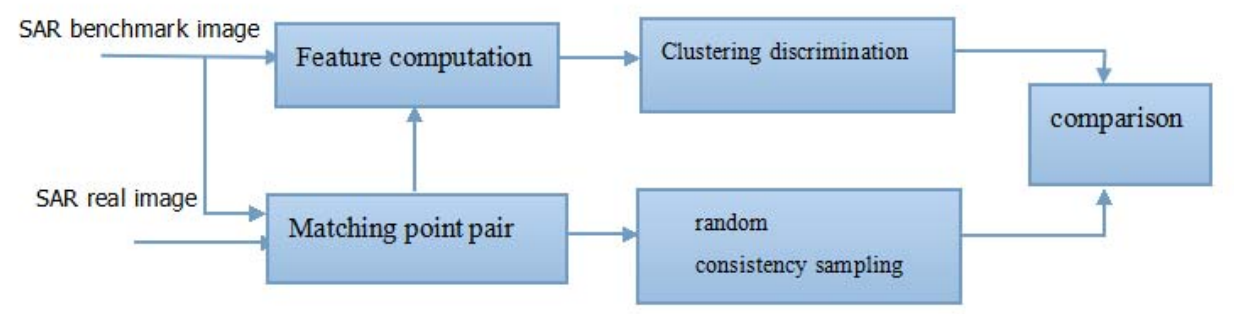

Figure 1. The matching point performance evaluation

\section{Predicting the fitment of matching point based on Random forest}

Predicting the fitment of matching point based on Random forest borrows the supervised learning framework in machine learning, it combines SAR image SIFT feature extraction algorithm , machine learning and scene matching algorithm. We use adaptive SAR image SIFT feature as the input, regarding benchmark image and real-time image matching points as the research object and then using the Random forest as classification, at last, the information of the fitment or unfitness of matching point is the output. The whole process as shown in figure 4.4, mainly including: (1).Generating the training set and test set; (2).classification training; (3).Predicting the fitment of the matching points.

The training set includes two parts: one is extracting SIFT point features in the image generated by training set, then, using the closest distance search matching points and computing the corresponding features; the other is obtaining the mark of the matching point is right or wrong, which using RANSAC marks the wrong points as 0 and marks the right points as 1 , respectively, negative samples and positive samples.

When obtaining the training set, we normalize every sample feature in it and regarding them as the input, then using Random forest algorithm to train the classification. The performance of the classifier measured by the predicting right rate of test set, repeatedly adjusting parameters and input features for classifier training and the test set until the performance of classification can be accepted. The test set generated process is similar to the training set, but only the area of scene which used to generate sample is different. 


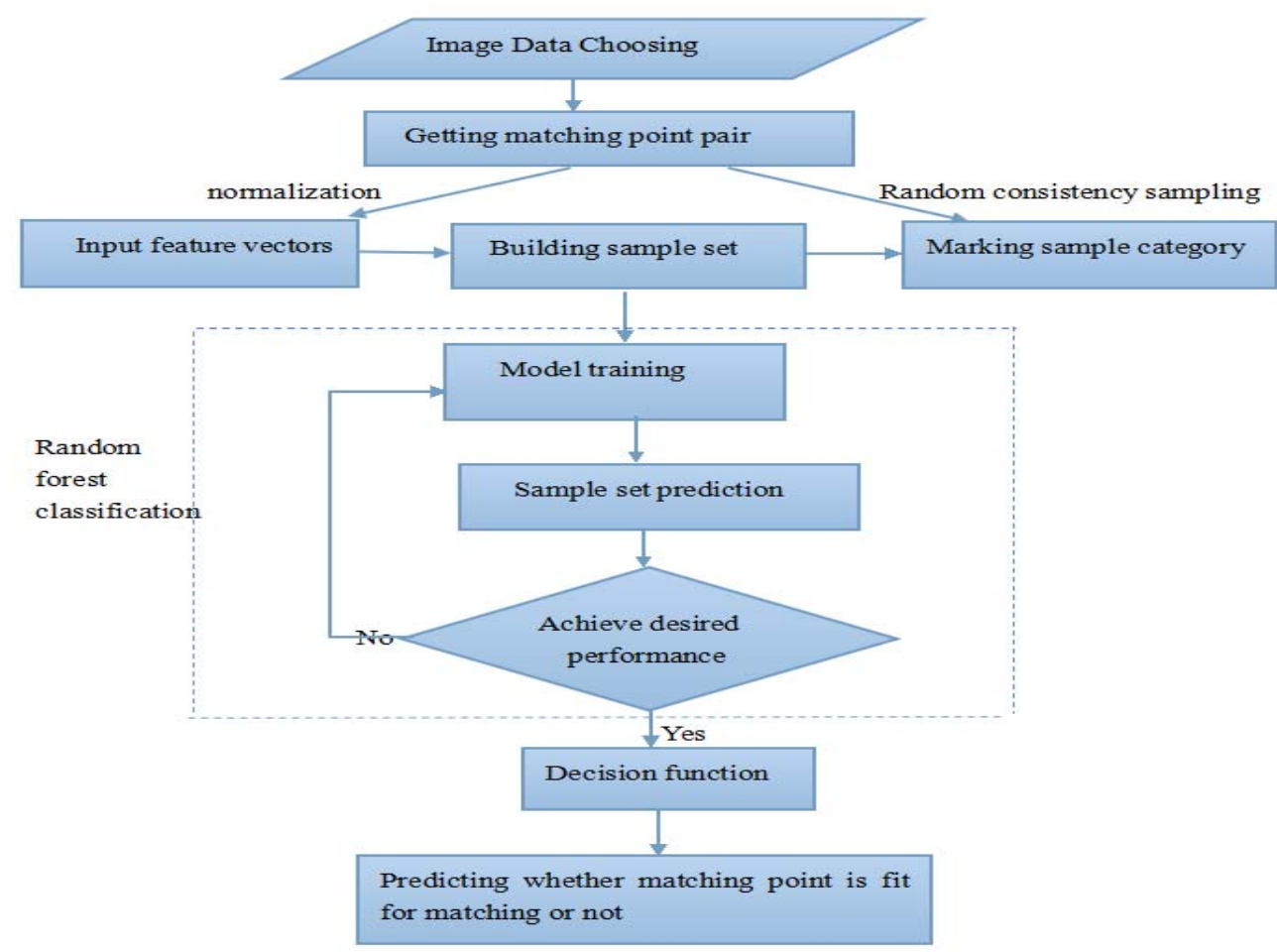

Figure 2. Predicting the fitment of matching point based on Random forest

We select $800 * 800$ benchmark image and $400 * 400$ real image, the number is 15 pairs, we compute matching points in every image pair. Extracting 500 right point pairs as the positive samples. As the number of wrong point pairs is 30 times of the right point pairs, in order to ensure the balance between positive and negative samples in the training set, we only extract 5000 wrong point pairs. The matching features include the closest distance, the ratio of the closest distance and the second closest distance, scale difference, matching points have same order or not and etc. Using random forest to train those sample and getting the last classification. Also, extracting 10 image pairs to obtain the test set in the similar way, and then using the training classification to predict the test set. The following table is the final classification result, the overall accuracy near $70 \%$, it shows that the right point pairs is 100 and the wrong point pairs are 3000 in the general experimental images, the correct rate is 3. 3\%. When using the above classification, it can eliminate $70 \%$ wrong matching and keep $70 \%$ right matching, so the right matching rate is up to $7.7 \%$, which can largely improves the RANSAC speed.

Table 1. the experimental results in this paper's classification algorithm

\begin{tabular}{lll}
\hline The test sample & Positive sample & Negative sample \\
$\begin{array}{l}\text { Random forest } \\
\text { discriminant result }\end{array}$ & 163 & 9330 \\
The miss rate & $32.9 \%$ & - \\
The virtual screening rate & - & $31.2 \%$ \\
The correct rate & $68.8 \%$ & - \\
\hline \hline
\end{tabular}

Figure 3 is the result of matching points in a subgraph of benchmark image. the marked green points are right matching points, the marked red points are wrong matching points. 


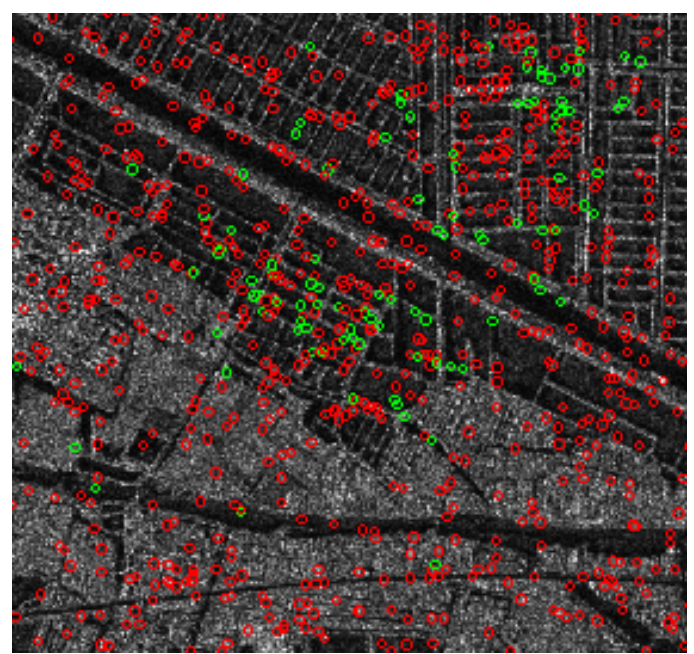

(a) matching result in actual

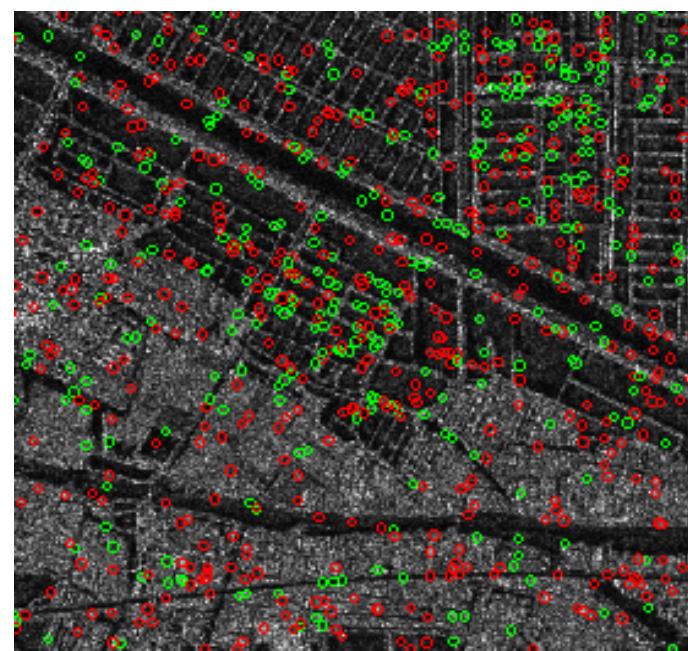

(b)matching result by classification prediction

Figure 3. Matching in actual and matching by classification prediction results

\section{Summary}

This paper mainly solve the problem that when using SIFT algorithm to finish scene matching, there are too many wrong matching points. The experiment shows that the algorithm can eliminate most wrong matching points and keep most of the right points.In this paper, the matching points on the discussion of screening is less involved in the previous predecessors' research, combined with machine learning method, can be used as a SAR image matching SIFT algorithm is an improved direction.

This work was supported by the National Natural Science Foundation of China under Grant 61273241 and 61273279.

\section{References}

[1] Dellinger F, Delon J, Gousseau Y, et al. SAR-SIFT: A SIFT-like algorithm for applications on SAR images[C], Geoscience and Remote Sensing Symposium (IGARSS), 2012 IEEE International. IEEE, 2012: 3478-3481.

[2] Fischler M A, Bolles R C.Random sample consensus: a paradigm for model fitting with applications to image analysis and automated cartography[J]. Communications of the ACM, 1981, 24(6): 381-395.

[3] Yi Z, Zhiguo C, Yang X.Multi-spectral remote image registration based on SIFT[J]. Electronics Letters, 2008, 44(2): 107-108.

[4] Bastanlar Y, Temizel A, Yardımc1 Y.Improved SIFT matching for image pairs with scale difference[J]. Electronics letters, 2010, 46(5): 346-348.

[5] Breiman L. Random forests[J]. Machine learning, 2001, 45(1): 5-32. 